\title{
EL DOMINIO DE RUBIANES EN EL SIGLO XIX: COMPOSICIÓN DEL MAYORAZGO Y DESVINCULACIÓN
}

\author{
Por \\ ANTONIO PRESEDO GARAZO ${ }^{1}$
}

Después de una serie de trabajos de cierta relevancia, se ha demostrado que la comprensión del desarrollo económico y social de Galicia pasa por un necesario estudio de los señoríos - tanto laicos como eclesiásticos-, ya que juegan un papel de primer orden en el acontecer histórico de dicha comunidad ${ }^{2}$. Partiendo del hecho constatado de que la mayor parte del dominio directo del terrazgo era poseído por estas instituciones, salta a la vista que el estudio de su composición, amén de su funcionamiento interno, nos pone en el camino correcto para acercarnos más verídicamente al conocimiento del pasado.

El presente trabajo pretende analizar la composición de un señorío laico - el de Rubianes - ligado inicialmente a la familia Gayoso, luego a los Ozores y finalmente a los Prado, situándolo en el languidecer del Antiguo Régimen, coincidiendo con las primeras manifestaciones del Liberalismo. El liberalismo económico parte de la premisa de que los indivi-

\footnotetext{
${ }^{1}$ Becado por la Consellería de Educación y Ordenación Universitaria de la Xunta de Galicia, Universidad de Santiago de Compostela.

${ }^{2}$ A. Villares (1982a y 1982b), P. Leiros (1986), P. Saavedra (1990), Ma J. Baz Vicente (1991), L. Domínguez Castro (1992) y V.M. Míguez Rodríguez (1993).

"CUADERNOS DE ESTUDIOS GALLEGOS", Tomo XLII, Fascículo 107, Santiago 1995.
} 
duos deben poseer de los medios de producción — entre ellos la tierra-, a partir de los cuales se generará riqueza. Y en el siglo XIX, la tierra es para el caso gallego en concreto, y para el conjunto del recién nacido Estado español, el medio de producción por excelencia. No es de extrañar, pues, que una de las primeras manifestaciones liberales del nuevo orden de cosas que se instaura con la Contemporaneidad sea el aniquilamiento del marco jurídico en el que se habían apoyado los señores del Antiguo Régimen para perpetuar su riqueza. El mayorazgo, y con él los bienes vinculados, antaño base de la riqueza para un grupo social situado en la cúspide de la sociedad estamental de Antiguo Régimen, se descontextualiza como consecuencia de la llegada del Liberalismo.

Un nada que nos adentremos en el estudio pormenorizado de lo que acontece en Galicia, vemos como la nobleza se adapta al nuevo orden de cosas. Se vive una paulatina transformación en las filas de la hidalguía rural - absentista o no-, que hace entrever la existencia de intereses determinados por parte de este grupo en el momento en que se producen los cambios ${ }^{3}$. Gracias al seguimiento detallado de lo que acontece con el Señorío de Rubianes hemos podido constatar lo dicho. El vaciado exaustivo de tres partijas y dos inventarios post mortem, así como otras noticias también procedentes de fuentes notariales, apuntan en esta dirección ${ }^{4}$.

\footnotetext{
${ }^{3}$ Para un seguimiento más exaustivo de los procesos de relevancia que caracterizan la Historia Agraria de Galicia en el siglo XIX son apropiados los trabajos de A. Artiaga et al. (1991) y X.R. Quintana (1991), y L. Domínguez Castro (1992), así como el de A. Bernárdez et al. (1993) para el estudio del comportamiento de una familia campesina gallega - los Quintero de San Juan de Carbia- a lo largo de la centuria decimonónica.

${ }^{4}$ En concreto, la documentación utilizada se halla actualmente en el Archivo Universitario de Santiago (AHUS), en la sección de Protocolos Notariales (PN). Se trata de la partija de los bienes de Don José Ramón Ozores Calo Romero, AHUS, PN, n 10.240, 1850, ff. 53-216. El inventario post mortem de los bienes de Doña María del Pilar Gayoso Gómez de los Cobos, AHUS, PN, $\mathrm{n}^{\circ} 10.242$, 1852, ff. 104-166. La partija de los bienes de Doña María Manuela Ozores y Balderrama, AHUS, PN, $n^{\circ} 10.248,1858$, ff. 139-158. La partija de los bienes de Doña Josefa Mosquera y Novales, AHUS, PN, $\mathrm{n}^{\circ} 10.249,1859$, ff. 64-100. Y el inventario post mortem de Doña María de la Concepción Ozores y Balderrama, AHUS, PN, $\mathrm{n}^{\circ} 10.254,1864$, ff. 3-49.
}

"CUADERNOS DE ESTUDIOS GALLEGOS", Tomo XLII, Fascículo 107, Santiago 1995. 


\section{ANÁLISIS SOCIOLÓGICO DE LA FAMILIA OZORES ${ }^{5}$}

En la partija que se hace de los bienes de Don José Ramón Ozores Calo Villafañe y Romero ${ }^{6}$, éste aparece citado como «señor de Rubianes» y casado con Doña María Aurora Balderrama. Sabemos que a la hora de su muerte residía en Santiago de Compostela con una buena parte de su familia, mientras el resto vivía en Madrid. Por lo tanto, un individuo determinado, un título, el año de su muerte - 1848- y el dato de que está viviendo en Santiago acompañado de algunos hijos. Diez años atrás —en 1838-, Don José era brigadier de Infantería y coronel del Cuartel Provincial de Compostela, tal como se especifica en el primer testamento que redactó su tía política Doña María del Pilar Gayoso de los Cobos ${ }^{7}$. Cuando la citada Doña María del Pilar dispone su definitiva manda testamentaria en 1851, expresa claramente su filiación con el brigadier a través de su esposo Don Miguel Gayoso de Mendoza, señor de Rubianes, muerto en 1837.

Comencemos por Don Miguel Gayoso y Mendoza, ya que es el primer señor de Rubianes que aparece en la documentación y está situado en el límite cronológico a partir del cual el señorío en cuestión se verá afectado por las leyes de desvinculación.

Sabemos que casó con la citada Doña María del Pilar Gayoso Gómez de los Cobos, hija de Don Domingo Gayoso Gómez de los Cobos - Marqués de Camarasa- y Doña Ana Gertrudis Bermúdez de Castro, y que ésta debía recibir anualmente de su hermano la cantidad de 11.000 duca$\operatorname{dos}^{8}$. Don Miguel muere en Coruña en 1837 sin descendencia de su matri-

\footnotetext{
${ }^{5}$ Vid. el recuadro genealógico de dicha familia expuesto en el Anexo I.

${ }^{6}$ AHUS, PN, $\mathrm{n}^{\circ} 10.240,1850$, ff. 53-216.

${ }^{7}$ Tal como se aprecia al leer su testamento redactado el 17 de enero de 1838: Declaro igualmente que con todo lo referido en las dos precedentes, me hallo en la mejor y mas amistad larmonia/ con el digno subcesor y familia de mi adorado señor difunto, el Brigadier de Ynfanteria y Coronel del Provincial de Compostela, Sr. Dn. Jose Ramon Ozores Calo Romero y Gayoso, a quien profeso particularmente un verdadero afecto, tanto por las relaciones que median de parentesco, como mucho mas aun si cabe, por las recomendables prendas morales que le distinguen, y pruebas que me ha dado y da de su buena correspondencia..., AHUS, PN, $\mathrm{n}^{\circ}$ 9.789, 1838, f. 21.

${ }^{8}$ El seguimiento genealógico hacia atrás de Doña María del Pilar Gayoso de los Cobos lo hemos realizado gracias a los datos aportados por J.S. Crespo (1958-85), situándola al lado de los Gayoso, que, iniciando sus actividades socioeconómicas en la Baja Edad Media, llegan a hacerse con los títulos - entre otros - de Condes de Amarante y Marqueses de Camarasa.
}

"CUADERNOS DE ESTUDIOS GALLEGOS", Tomo XLII, Fascículo 107, Santiago 1995. 
monio con Doña Pilar. Su lugar de residencia no coincide con el núcleo en el que se hallan la mayor parte de sus bienes: Vilagarcía de Arousa. Es decir, nos hallamos ante un noble absentista más. Un noble absentista que muere sin descendencia legítima directa. $Y$ es precisamente esta especial circunstancia la que hace que aquel brigadier conocido por nosotros, sobrino suyo, se introduzca en los intereses del Señorío de Rubianes.

La señora viuda de Rubianes - Doña María del Pilar - redacta un año después de la muerte de su esposo dos escrituras de relevancia que especifican en qué condiciones habrá de pasar el Señorío de Rubianes a la siguiente generación, acuñando los presupuestos que dan forma a las mandas testamentarias del difunto Don Miguel, objeto de interés en la tercera parte de este estudio.

Nos introducimos, así, en la segunda generación con Don José Ramón Ozores - además de brigadier, señor de la Casa de Rial —, quien a partir de 1837 se convierte en señor de Rubianes, doce años antes de su muerte. Reside en Santiago junto a su mujer Doña María Aurora Balderrama y Barrio. De su matrimonio nacen once hijos: cinco varones y seis mujeres 9 . De Doña María Aurora apenas sabemos que fue beneficiada por dos de sus hijas ${ }^{10}$, además de una venta que hizo como apoderada de su marido y su hijo Don Jacobo en $1838^{11}$. Cuando muere Don José Ramón, tan sólo residían en Santiago tres de sus once hijos: Doña Luisa - casada con Don José Rodríguez Galdo-, Doña María de la Concepción —-soltera-, y Doña Ramona — casada con Don José María de Prado y Neyra, marqués de San Martiño de Hombreiro ${ }^{12}$ - . Salvo Doña Antonia, que reside en Coruña con su marido Don Andrés Somoza Monriu y Castro, el resto de los hermanos viven en la capital del Estado. El llamado a suceder en el vínculo a Don José Ramón — su primogénito Don Juan María-, después de enviudar en 1835, aparece en Madrid como senador. Por su parte, Don José Javier es doctor en jurisprudencia, Don Joaquín teniente coronel de Infantería y oficial del Ministerio de Guerra, y Don Eduardo llega a segundo comandante de Infantería y capitán de Artillería. Doña María de

\footnotetext{
${ }^{9}$ Uno de ellos - Don Luis- muerto en pupila edad.

${ }^{10}$ Por una parte, Doña María Manuela, de quien conocemos la partija de sus bienes; y de otra, Doña María de la Concepción, de la que conocemos su inventario post mortem.

${ }^{11}$ AHUS, PN, nº 9.789, 1838, ff. 104-105.

${ }_{12}$ Para un seguimiento más pormenorizado del Marquesado de San Martiño de Hombreiro, es imprescindible la tesis de licenciatura de V. Míguez Rodríguez (1993).
}

"CUADERNOS DE ESTUDIOS GALLEGOS", Tomo XLII, Fascículo 107, Santiago 1995. 
los Dolores también emparenta a la familia con el Ejército tras su matrimonio con el coronel de Infantería y primer comandante del Quinto Regimiento de Artillería, Don Fernando Camus. Doña María Manuela morirá soltera.

Don Juan Ozores y Balderrama aglutina los intereses de la familia en la tercera generación. Señor de Rubianes después de la muerte de su padre en 1849, toma por esposa a la marquesa de Aranda y Gimarey, Doña Josefa Mosquera y Novales. En 1835, Don Juan María enviuda quedando consigo cuatro hijos. De ellos, el primogénito, Don Jacobo Ozores y Mosquera, es quien hereda el título de marqués de Aranda y Gimarey tras la muerte de su madre, siendo también vizconde de Quintanilla a partir de 1859. Es el único de los cuatro hermanos que aparece afincado en Madrid, ya que sus tres hermanas lo están en Santiago. Doña María del Carmen casa con Don Vicente Calderón y Oreyro — conde de San Juan—; Doña Felisa no casa; y Doña Concepción se desposa con su primo Don José María de Prado y Ozores - marqués de san Martiño de Hombreiro-.

Ya tenemos definidas las tres generaciones objeto de estudio que poseyeron el título de señor de Rubianes. Los protocolos notariales de Don Francisco Antonio Vieites no nos permiten avanzar más. Pero es suficiente. Tenemos a nuestro alcance algunos elementos de juicio imprescindibles para valorar los principales comportamientos del grupo familiar.

Lo primero que llama la atención es que ninguno de los que se sitúan al frente del vínculo reside en Rubianes, sito en la Ría de Arousa. El primero de ellos —Don Miguel — vivía en Coruña, y su mujer —Doña María del Pilar-, pese a haber hecho importantes inversiones en la restauración de la casa-pazo de Rubianes ${ }^{13}$, reside en Santiago. Don José Ramón también vive en la ciudad compostelana. Don Juan María y su hijo primogénito Don Jacobo prefieren Madrid. Evidentemente, el absentismo les obliga a administrar los bienes a través de mayordomos encargados de llevar las cuentas, no sólo en Rubianes, sino también en el resto de los bienes que $\operatorname{poseen}^{14}$.

\footnotetext{
${ }^{13}$ AHUS, PN, $n^{\circ} 9.789,1858$, f. 27.

${ }^{14} \mathrm{El}$ absentismo es un hecho común a otros señoríos hidalgos gallegos del mismo momento histórico. De hecho, así se constata en la partija del señor de un vínculo sitio en el ayuntamiento lucense de Láncara, Don Salvador María Quiroga y Puga, residente en Madrid en 1848 al ser diputado en Cortes, además de magistrado en la Audiencia de Albacete; mientras que su mujer residía en Santiago. AHUS, PN, n ${ }^{\circ} 10.238,1848$, ff. 77
}

"CUADERNOS DE ESTUDIOS GALLEGOS", Tomo XLII, Fascículo 107, Santiago 1995. 
Otro elemento de notable relevancia es el análisis de las distintas profesiones a que se dedican los distintos miembros de la familia, interesándonos principalmente el paradero de los segundones, y por extensión, el de todos aquellos que de partida no están llamados a ocupar el puesto de preferencia que desde el Antiguo Régimen se les venía reservando a los primogénitos. Son tres las salidas escogidas. La primera de ellas es el oficio de armas, ocupando puestos de importancia en el ejército. Acordémonos que Don José Ramón fue antes brigadier de Infantería que señor de Rubianes, y que dos de sus hijos siguieron su mismo camino: Don Joaquín y Don Eduardo. Luego está la vía de la política, y aquí no debemos olvidarnos de Don Juan María, que fue senador a la vez que señor de Rubianes. Y tercer lugar, la carrera de Derecho, siendo de ello claro representante Don José Javier, que es doctor en jurisprudencia. Una amalgama de nuevas ocupaciones para un grupo social privilegiado en el Antiguo Régimen, que ahora intenta integrarse en el nuevo orden de cosas derivado del proceso de afianzamiento del Liberalismo ${ }^{15}$.

\section{DESCRIPCIÓN PORMENORIZADA DEL SEÑORÍO DE RUBIANES EN 1830}

Nos hallamos frente a un grupo social que se articula a partir de una realidad determinada. El hecho de que los señores de Rubianes actúen

172. En otra investigación que estamos realizando, el cura Don Juan de Quiroga Riomol, apoderado y mayordomo a partir de la década de 1860 de la Casa de Goyán, le compra a su señor -Don Manuel Souto Freire - residente en la capital lucense, todas las rentas que dicha casa recibía en el Partido de Caurel. Cuando un hidalgo era señor de varias casas, forzosamente tenía que vivir lejos de los solares, y en el s. XIX, con las ocupaciones profesionales y políticas, el absentismo no se acentuó. Cobra importancia en este contexto, la idea de que la no residencia de los antiguos señores en los núcleos de sus patrominios permite en ciertos casos el ascenso social de los mayordomos en el seno de la comunidad campesina a lo largo del siglo XIX. Por otra parte, no se trata de una característica exclusiva de la nobleza gallega, tal como demuestra B. Yun Casalilla (1991) al estudiar el marquesado castellano de Cuéllar.

${ }^{15}$ Para esta nueva ubicación a nivel profesional de la hidalguía gallega, puede verse L. Domínguez Castro (1992), pp. 21-22, para la comarca del Ribeiro. A. García Sanz (1991) también la constata al estudiar el marquesado de Lozoya, demostrando que además de la renta - evidentemente, la fuente de ingresos más segura-, los marqueses son conscientes de los buenos sueldos que se cobran ejerciendo una buena profesión liberal. 
siguiendo unos patrones de comportamiento específicos, nos está indicando su implicación dentro de un orden de cosas en el cual todos los elementos del conjunto son imprescindibles para su entendimiento. Para estudiar como se produce la desarticulación del Señorío de Rubianes, estamos obligados precedentemente a analizar el patriminio en sí mismo. Difícilmente podemos hablar de desvinculación si no existen previamente vínculos.

\section{Los bienes sujetos al vínculo}

Son cinco las administraciones que componen los bienes vinculares del señorío que nos ocupa. Vayamos una a una.

* La Administración de Rubianes centralizaba los bienes y rentas poseídos por el señorío en los ayuntamientos de Caldas de Reis, Moraña, Rianxo, Vilagarcía de Arousa y Vilanova de Arousa. Tras la suma de los distintos valores en que aparecen tasados la casa-pazo, el jardín, la casa de labranza, el mobiliario y la ropa, la cantidad total asciende a 92.129 Rs.

Respecto a la dedicación del terrazgo, hemos hecho una triple distinción entre labradío, monte y viñedo, pues de dicha tipología se derivan tanto distintos rendimientos agrarios como diferentes usos. El resultado total es de 79,29 Has., cuyos porcentajes son expresados en la gráfica 1.

\section{Gráfica 1. Distribución porcentual de los terrenos que componen la Administración de Rubianes.}

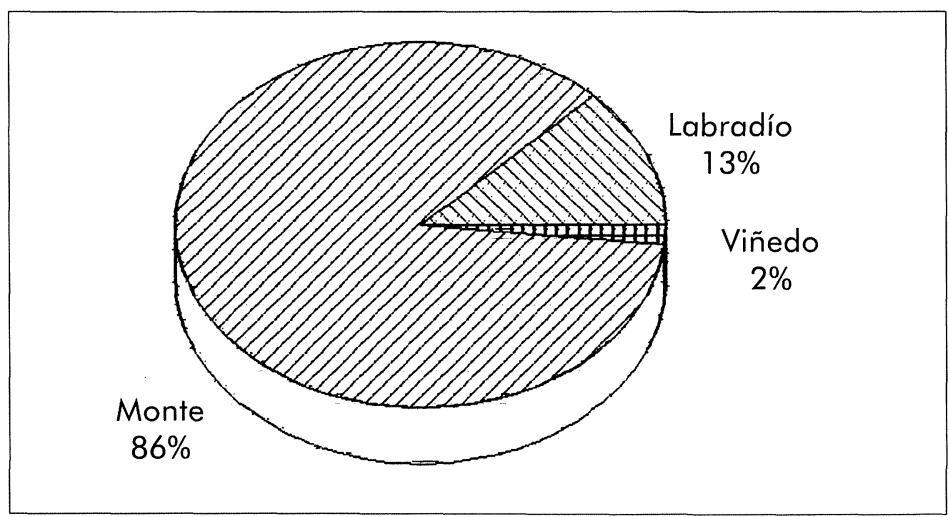

Salta a la vista por su contundencia, la importante extensión ocupada por el monte - 63,665 Has.- , que llega a suponer 5,95 veces más que la cifra resultante de la suma de las hectáreas dedicadas a labradío y viñe-

"CUADERNOS DE ESTUdiOS GALLEGOS", Tomo XLII, Fascículo 107, Santiago 1995. 
do conjuntamente ${ }^{16}$.

La renta obtenida de los campesinos que trabajan tierras sobre las que los señores de Rubianes tienen derecho a percibirla, puede verse en la tabla 1.

Tabla 1. Renta recibida en la Administración de Rubianes.

\begin{tabular}{lrcrrrr}
\hline & Caldas & Moraña & Rianxo & Vilagarcía & Vilanova & Total \\
\hline Trigo (Hls.) & 11,02 & 9,47 & & 85,84 & 0,93 & 104,44 \\
Centeno(Hls.) & 0,31 & & & 41,59 & 0,15 & 42,05 \\
Maíz(Hls.) & & & & 1,46 & 10,01 & 11,47 \\
Mijo(Hls.) & & & & 20,40 & 6,54 & 26,94 \\
Cebada(Hls.) & 9,11 & \multirow{2}{*}{9,47} & & 70,26 & & 88,84 \\
Vino(Hls.) & & & & 16,41 & & 14,41 \\
Dinero(Rs.) & 33 & & 275 & 4.286 & & 4.594 \\
\hline
\end{tabular}

Hay dos datos de interés tocantes a la composición de dichas rentas ${ }^{17}$. Por una parte, se aprecia una variabilidad en los productos cobrados, también aplicable a los ayuntamientos en que se cobran, evidente si comparamos el caso de Rianxo - donde tan sólo se reciben 275 Rs.- con Moraña -en el que se reciben 18,94 Hls. de cereal, sumando el trigo y la cebada, sin contar las seis gallinas aquí recibidas-. El otro elemento a destacar es el distinto porcentaje de cereales recibidos, sumando un total de 293,04 Hls. que, expresados porcentualmente demuestran un predominio absoluto en favor del trigo, seguido por la cebada, el centeno y el mijo, mientras que el maíz no deja de ser un elemento meramente anecdótico, tal como se puede apreciar en la gráfica 2 .

\footnotetext{
${ }^{16}$ La importancia porcentual de la superficie dedicada a monte no nos debe asustar, sobre todo si tenemos en cuenta los estudios realizados por A. Bouhier (1979), P. Saavedra (1982 y 1989) y X. Balboa (1990), en los que se destaca la importancia ocupada por el monte en las estructuras productivas de la economía campesina gallega.

${ }^{17}$ Un seguimiento pormenorizado de la renta en el siglo XIX en Galicia puede verse en R. Villares (1982b) y A. Artiaga (1984); en Cataluña, R. Congost (1990); y para Mallorca, J. Suau Puig (1991).
}

"CUADERNOS DE ESTUDIOS GALLEGOS", Tomo XLII, Fascículo 107, Santiago 1995. 


\section{Gráfica 2. Distribución porcentual de la renta ceralícola cobrada en la Administración de Rubianes}

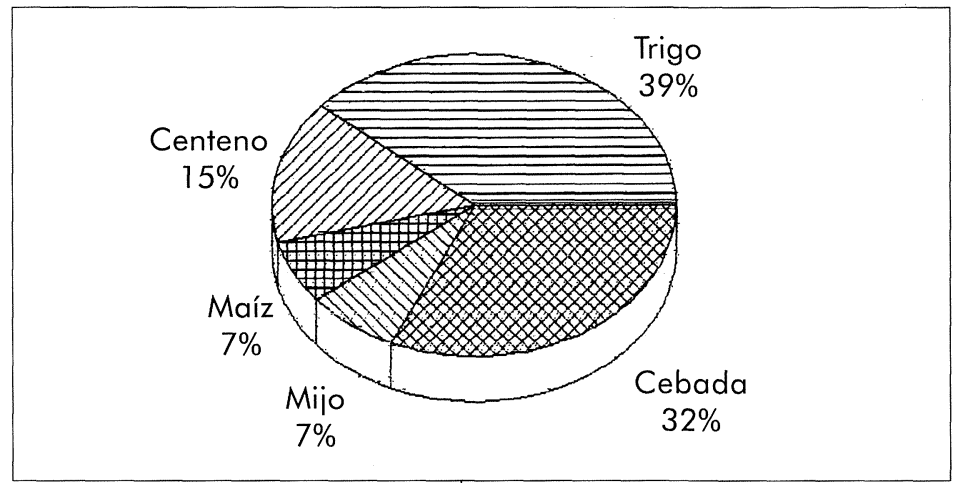

A esta administración estaban sujetas una serie de pensiones de carácter irrelevante, si tenemos en cuenta la ganancia rentística que obtenía la Casa; mas no por eso hay que discriminar el dato: se trata de $1,55 \mathrm{Hls}$. de trigo, 4,36 Hls. de centeno, $1,55 \mathrm{Hls}$. de cebada y 35 Rs. y 24 mrs. ${ }^{18}$.

* Más relevancia adquieren los bienes en pleno dominio en la Administración de Badajoz, donde el señorío de Rubianes poseía a través de la casa de Mendoza, una extensión de 6.936 cabezas de tierra a dehesa que producían anualmente en renta 43.467 Rs., sujetas al pago de 10.682 Rs. y 9 mrs. en concepto de pensiones ${ }^{19}$.

* En la Administración de Guntín y Castelo dos Infantes, centrada en los ayuntamientos lucenses de Sarria, Bóveda y Póboa de Brollón, una

${ }^{18}$ Por ejemplo, se le pagaba al marqués de Vilagarcía cierta cantidad, tal como se especifica cuando se dice que Al Sr. Marques de Villagarcia se pagan veinte y cuatro ferrados de centeno, ocho de mediado y cuatro reales por el lugar do Pazo y mas bienes en Ruvianes..., AHUS, PN, $\mathrm{n}^{\circ} 10.242,1852$, f. 139.

${ }^{19}$ Se trata de una serie de cargas anuales que debía pagar el señor para liquidar una serie de préstamos que le fueron concedidos anteriormente, y de los que todavía se debe cierta cantidad, amén de gastos para la lucha contra las plagas de langosta: Ydem al Sr. Marques de Cusano, vecino de Madrid, por un censo de cuarenta y cuatro mil setecientos y tres reales y cinco mrs. a tres por ciento impuesto sobre los citados bienes, mil trescientos cuarenta y dos reales y nueve mrs.

Se considera como pension los gastos indespensables que ocasiona el reconocimiento pericial de la langosta, importantes por quinquenio ochenta y cinco mil reales cada año. AHUS, PN, nº 10.240, 1850, f. 190

"CUADERNOS DE ESTUDIOS GALLEGOS", Tomo XLII, Fascículo 107, Santiago 1995. 
parte de los bienes que posee el señorío en pleno dominio - existentes únicamente en Sarria y Bóveda-, aparece valorada en función de lo que rinden tras ser cedidos en arrendamiento a cambio del pago de una renta. De este modo, la cesión de dos casas, una huerta y seis prados, produce anualmente 1.417 Rs. y 1,58 Hls. de centeno.

En lo que toca al terrazgo, se aprecian cambios cualitativos a tener en cuenta si comparamos esta administración con la de Rubianes. Se registra un descenso acentuadísimo en favor de las 79,29 Has. de Rubianes, frente a las 8,89 Has. que ofrecen Guntín y Castelo dos Infantes. Aunque la extensión dedicada a monte ocupa de nuevo un lugar destacado, el primer puesto les corresponde a las tierras dedicadas a viñedo, tal como refleja la gráfica 3.

\section{Gráfica 3. Distrubición porcentual de los terrenos que componen la Administración de Guntín y Castelo dos Infantes}

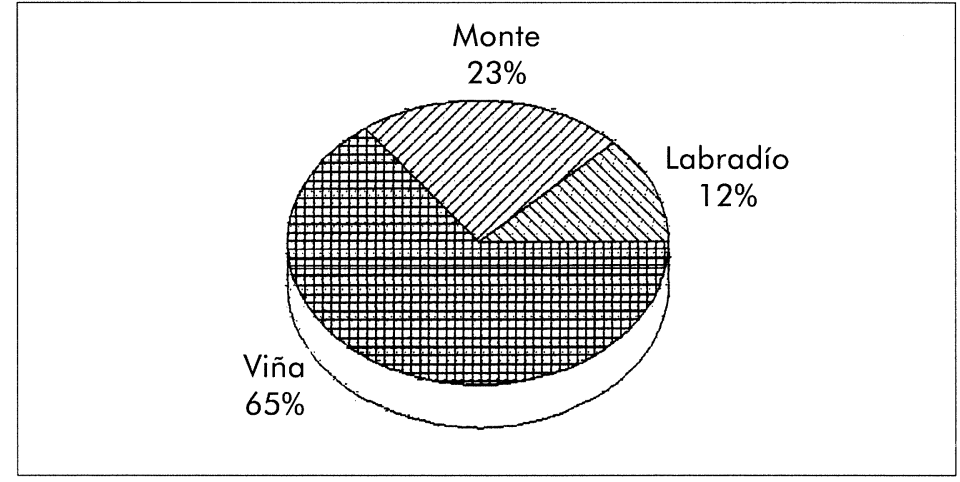

El análisis de las rentas forales también experimenta ciertos cambios a tenor de lo que se observa en la tabla 2.

Tabla 2. Renta recibida en la Administración de Guntín y Castelo dos Infantes

\begin{tabular}{lrrcr}
\hline & Sarria & Bóveda & Póboa de Brollón & Total \\
\hline Trigo (Hls.) & 28,11 & 3,30 & 9,27 & 40,68 \\
Centeno (Hls.) & 144,14 & 22,72 & 39,06 & 205,92 \\
Vino (Hls.) & & 9,83 & 30,62 & 40,44 \\
Dinero (Rs.) & 137 & 130 & 3 & 270 \\
\hline
\end{tabular}

"CUADERNOS DE ESTUdiOS GALLEGOS", Tomo XLII, Fascículo 107, Santiago 1995. 
En el seguimiento particular del cereal recibido, es evidente el predominio abrumador del centeno, como de hecho lo demuestran los 205,92 Hls. cobrados anualmente, frente a los 40,68 Hls. procedentes de la renta expresada en trigo.

El Señorío de Rubianes debía de pagar cada año en esta administración, en concepto de pensión, 58 Rs. y 28 mrs. a la Hacienda Nacional en representación del suprimido Monasterio de Sarria... por los bienes de la Casa del Castillo... ${ }^{20}$ Una deuda contraída con Hacienda en la órbita del proceso desamortizador de Mendizábal; lo cual indica que los Gayoso invierten en la compra de bienes eclesiásticos nacionalizados.

* La Administración de Villafranca del Bierzo produce al año 2.500 Rs. y 21 cuartales de centeno derivados de la cesión en arriendo de una casa, una huerta y una viña. Además, 467 Rs. y 23 cuartales procedentes de rentas forales.

* Finalmente, está la Administración de Lugo, caracterizada por la preponderancia de las rentas forales frente a la falta de bienes en pleno dominio. Los datos susceptibles de análisis se reflejan en la tabla 3.

Tabla 3. Renta recibida en la Administración de Lugo

\begin{tabular}{lrccr}
\hline & Lugo & Outeiro de Rei & Pol & Total \\
\hline Trigo(Hls.) & 10,24 & 5,14 & 0,16 & 15,54 \\
Centeno(Hls.) & 123,42 & 23,76 & 7,37 & 154,55 \\
Dinero(Rs.) & 2.744 & & & 2.744 \\
\hline
\end{tabular}

Añadámosle otro tipo de rentas de carácter más variado: $0,26 \mathrm{Hls}$. de castañas, 16 docenas de anguilas, 1 carro de leña y veinte panecillos ${ }^{21}$.

Se repiten los mismos resultados que constatamos en la Administración de Guntín y Castelo dos Infantes. Una disminución proporcional del cereal en beneficio de otros productos. $\mathrm{Y}$ dentro del mismo cereal, un predominio abrumador del centeno sobre el trigo, alcanzando en este caso la proporción de 9,9 Hls. de centeno por cada uno de trigo, superior a la relación de cinco a uno registrado para Guntín y Castelo dos Infantes.

\footnotetext{
${ }^{20}$ AHUS, PN, $\mathrm{n}^{\circ} 10.240,1850$, f. 152.

${ }^{21}$ Este carácter mixto de la renta ha sido destacado para el concejo asturiano de Caravia como feudalizante por J.M. Rodríguez de la Huerta (1992).
}

"CUADERNOS DE ESTUDIOS GALLEGOS", Tomo XLII, Fascículo 107, Santiago 1995. 


\section{Los añadidos}

El patrimonio familar hasta aquí descrito, se irá redondeando con otros bienes que se anexan al dominio. El origen de los mismos responde a una doble explicación. Las adquisiciones de bienes raíces y rentas que hacen los señores de Rubianes a lo largo del período de tiempo estudiado, incrementan las ganancias anuales recibidas, pero no inflan el conjunto total de posesiones sujetas al mayorazgo. Debemos de situar en esta tipología las inversiones destacadas más atrás, al referirnos a la compra de bienes nacionalizados con la desamortización de Mendizábal.

La otra vía es una clara herencia del Antiguo Régimen: la incorporación de distintos patrimonios debido a las estrategias familiares puestas en funcionamiento cuando los miembros de la familia se casan, o son objeto del beneficio de un testamento redactado a su favor.

Así, cuando en 1838 recae sobre Don José Ramón Ozores el título de señor de Rubianes, son incorporados al dominio en cuestión los bienes sobre los que tenía derechos hereditarios. Nos referimos a las Casas de Luaces, de Ozores y de Calo Romero. Veamos a que añadidos nos referimos.

Don Miguel Gayoso de Mendoza compra para su mujer una serie de terrenos adyacentes a los ocupados geográficamente por la Administración de Rubianes: 1,05 Has. de labradío y 2,98 Has. de monte. Su mujer -Doña María del Pilar Gayoso - aporta una serie de rentas. En el ayuntamiento de Moraña percibe $8,13 \mathrm{Hls}$. de maíz, derivados de contratos de arrendamiento. En la Administración de Noia las rentas vuelven a ser forales, observándose de nuevo un claro predominio del centeno, con una proporción de 7,97 Hls. por cada uno de trigo. Una descripción más pormenorizada la podemos observar en la tabla 4.

Tabla 4. Renta recibida en la Administración de Noia

\begin{tabular}{lrrrr}
\hline & Noia & Lousame & Porto do Son & Total \\
\hline Trigo (Hls.) & 1,93 & & 3,55 & 5,48 \\
Centeno (Hls.) & 1,45 & 11,30 & 31,00 & 43,75 \\
Dinero (Rs.) & 1.012 & 18 & 8 & 1.038 \\
\hline
\end{tabular}


La misma Doña María del Pilar aporta 39.327 Rs. derivados de la valoración de siete casas, el mobiliario, las joyas, la loza... encontrados en ellas, además de un horno, sito todo ello en Santiago.

Don José Ramón Ozores incorpora en su persona una serie de bienes procedentes de distintas herencias con las que fue beneficiado, tal como se ha dicho. Como señor de Luaces, centra en su persona cuatro administraciones: la de Zamora, la de Santiago de Compostela, la de Padrón y la de Pontevedra.

Los bienes sitos en Zamora - sobre los que tiene pleno dominio- son expresados en la partija a partir de lo que producen en renta tras su arrendamiento $^{22}$ : 163 fanegas de trigo, 163 fanegas de cebada, 36.788 Rs. y 2 carros de paja. Estas rentas son complementadas anualmente por otra serie de cargas recibidas por foros y censos: 2 fanegas y 6 celemines de trigo, 2 fanegas y 6 celemines de cebada y 1.486 Rs. y $36 \mathrm{mrs}$. Es evidente, pues, que los principales ingresos que recibe el señor de Luaces - que lo es también de Rubianes - en la Administración de Zamora proceden fundamentalmente del arrendamiento de sus bienes en pleno dominio, y no de foros.

En Galicia, los resultados cambian considerablemente. La Casa de Luaces sólo posee en la Administración de Santiago bienes en pleno dominio, también valorados a partir de lo que producen en renta procedente de arrendamientos: 60,02 Hls. de trigo, 2.153 Rs. y $31 \mathrm{mrs}$., 17 gallinas, 9 carros de leña y otro más de abono. La tabla 5 expresa lo que acontece con las rentas forales.

Es obvio el predominio de la renta en trigo, seguido en segundo lugar por el centeno. Tanto el maíz como el mijo, o mismamente la cebada, son meros elementos anecdóticos.

Como herencia procedente de la Casa de Ozores, Don José Ramón posee las Administraciones de Cambados y Tui. La primera de las dos presenta una panorámica muy similar a lo que acontece en Santiago. Los bienes en pleno dominio también se arrendan, dándose el predominio de

${ }^{22}$ Tal como se ha visto antes con Badajoz y Villafranca del Bierzo, predominan las cesiones a partir de arrendamientos en los contratos agrarios que el Señorío de Rubianes lleva a cabo para la obtención de una renta más flexible que la derivada del foro. En el marquesado castellano de Lozoya, los contratos de arrendamiento son la clave para la obtención de rentas, tal como demuestra A. García Sanz (1991).

"CUADERNOS DE ESTUdIOS GALLEGOS", Tomo XLII, Fascículo 107, Santiago 1995. 
Tabla 5. Renta foral percibida por la Casa de Luaces en Galicia

\begin{tabular}{lrccr}
\hline & Santiago & Padrón & Pontevedra & Total \\
\hline Trigo (Hls.) & 58,54 & & 9,11 & 67,65 \\
Centeno (Hls.) & 42,22 & & & 42,22 \\
Maíz (Hls.) & 1,46 & & & 1,46 \\
Mijo (Hls.) & 3,01 & & 9,11 & 9,01 \\
Cebada (Hls.) & & & 424 & 1.892 \\
Dinero (Rs.) & 631 & 837 & & \\
\hline
\end{tabular}

la renta cerealícola cobrada en maíz ${ }^{23}$. Respecto a la renta foral - salvo 0,6 Hls. de vino, 79,5 gallinas y 740 Rs. y 3 mrs.- el cereal preferido como canon rendístico vuelve a ser el trigo, tal como se especifica en la gráfica 4.

\section{Gráfica 4. Distribución porcentual de la renta ceralícola foral cobrada en la Administración de Cambados}

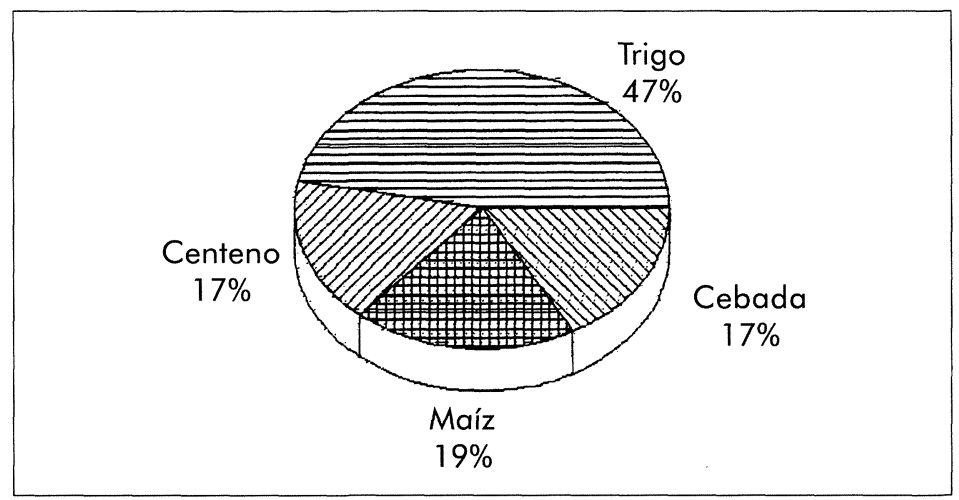

En la Administración de Tui, donde sólo se perciben rentas forales, el mijo se destaca como cereal ideal del que la Casa de Ozores recibe más hectolitros de renta anualmente - 34,97-, llegando a suponer la mitad de las rentas cobradas en cereal en dicha administración, tal como se puede ver en la gráfica 5 .

${ }^{23}$ Son cobrados anualmente 3,81 Hls. de trigo, 6,07 Hls. de centeno, 65,29 de maíz y 0,15 de cebada, 319 Rs. e 7 mrs.

"CUADERNOS DE ESTUDIOS GALLEGOS", Tomo XLII, Fascículo 107, Santiago 1995. 
Gráfica 5. Distribución porcentual de la renta ceralícola cobrada en la Administración de Tui

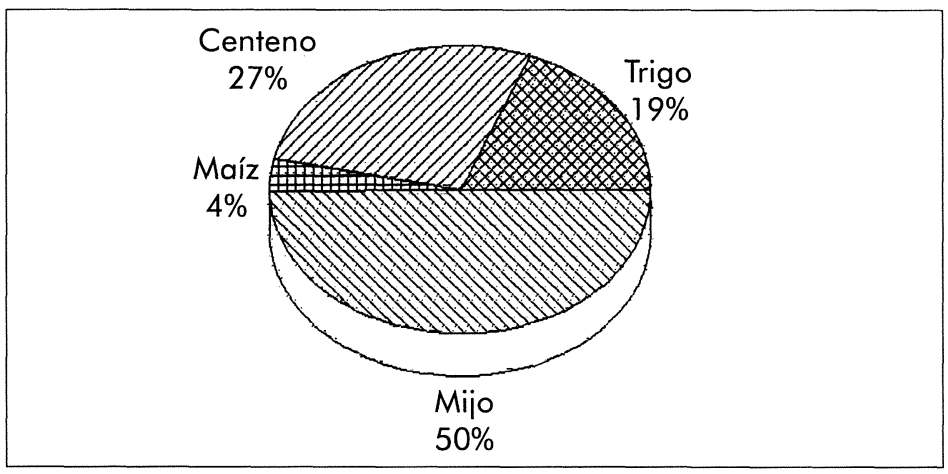

Don José Ramón también añade al Señorío de Rubianes los bienes que hereda procedentes de la Casa de Calo y Romero, centrados en una administración que engloba los bienes sitos en los partidos judiciales de $\mathrm{Mu}-$ ros, Corcubión, Negreira y Carballo. De los bienes en pleno dominio, recibe anualmente por el arriendo de cinco casas, dos piezas de monte, y por los cubiertos que se ponen en el campo de la feria de Berdillo ${ }^{24}, 1.161$ Rs. y $0,96 \mathrm{Hls}$. de trigo. De los distintos porcentajes de cereales procedentes del cobro de rentas forales y censos, nos habla la gráfica 6 :

Gráfica 6. Distribución porcentual de la renta foral cerealícola cobrada por la Casa de Calo y Romero

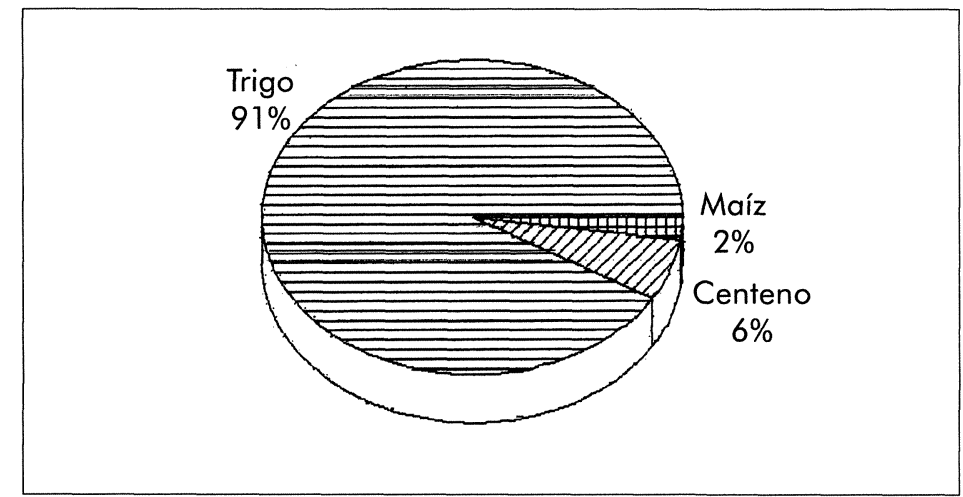

${ }^{24}$ AHUS, PN, $\mathrm{n}^{\circ} 10.240,1850$, f. 120.

"CUADERNOS DE ESTUDIOS GALLEGOS", Tomo XLII, Fascículo 107, Santiago 1995. 
Se aprecia un dominio absoluto de la renta percibida en trigo, que supone 10,4 veces los 43,057 Hls. que suman en conjunto el centeno y el maíz.

La marquesa de Aranda y Guimarey - Doña Josefa Mosquera y Novales-, esposa de Don Juan María Ozores y Balderrama, debe ser destacada por los bienes que aporta a su matrimonio con el señor de Rubianes, situados únicamente en Madrid y que serán divididos en la partija que se hizo en 1835 después de su fallecimiento. Se trata de 5 casas valoradas en 1.702.764 Rs., un número indeterminado de fincas con una extensión de 217 fanegas, 95 celemines y 16,5 cuartillos, 43.000 Rs. de crédito dados a su favor, 19.956 Rs. procedentes de fincas vendidas, 501.233 Rs. invertidos en deuda del Estado, y 62.862 Rs. derivados de la participación de la marquesa en negocios y corporaciones.

\section{Una valoración global del dominio}

Vistos pormenorizadamente cada uno de los elementos que a nivel patrimonial están definiendo el monto total de los bienes del dominio, no está de menos valorarlos conjuntamente. Se aprecian diferencias evidentes entre los bienes pertenecientes a esta casa hidalga en Castilla y en Galicia. La mayor parte de los situados en Castilla son poseídos a través del directo dominio, de ahí que se arrienden en la mayor parte de los casos. En Galicia, por el contrario, los bienes raíces parecen ser menos, produciendo una renta mayor que aquéllos de los que se posee el útil, que a su vez son aforados. No existe una regularidad en la calidad de las tierras que conforman las distintas administraciones del dominio. En todo caso, el labradío nunca se destaca como mayoritario en ninguna administración. Recordemos que la superficie ocupada por el monte en la Administración de Rubianes supone el $86 \%$ del total, y que en la Administración de Guntín el viñedo ocupa el $65 \%$.

La tabla 6 nos servirá para un seguimiento más detallado de la renta, así como el mapa adjunto (Pag. 86) para una mejor localización geográfica de las distintas administraciones en Galicia.

Se constatan tres datos de relevancia. No existe una homogeneidad a la hora de cobrar la renta en las distintas administraciones. En caso de coincidir dos o más en el cereal principalmente cobrado, no por ello se da una correspondencia entre el monto total recibido y el resto de las otras calida- 
Tabla 6. Renta anual recibida en 1830 por el dominio de Rubianes procedente de todas sus administraciones —en Hls. y Rs.-

\begin{tabular}{lrrrrrrr}
\hline Administración & Trigo & Centeno & Maíz & Mijo & Cebada & Vino & Dinero \\
\hline Rubianes & 107,44 & 42,05 & 19,60 & 26,94 & 88,84 & 14,41 & 4.594 \\
Badajoz & & & & & & & 43.467 \\
Guntín & 40,68 & 207,50 & & & & 40,44 & 1.687 \\
Villafranca & & & & & & & 2.967 \\
Lugo & 15,54 & 154,55 & & & & & 2.744 \\
Noia & 5,48 & 43,75 & & & & & 1.038 \\
Zamora & & & & & & & 38.275 \\
Santiago & 111,56 & 42,22 & 1,46 & 3,01 & & & 2.784 \\
Padrón & & & & & & & \\
Pontevedra & 9,11 & & & & 9,11 & \\
Cambados & 57,79 & 25,46 & 95,11 & & 19,78 & 0,6 & 1.059 \\
Tui & 13,32 & 19,26 & 3,38 & 34,97 & & & 229 \\
Muros & 413,86 & 29,26 & 13,78 & & & & 4.486 \\
\hline Total & 774,78 & 564,05 & 133,33 & 64,92 & 117,73 & 55,45 & 104.593 \\
\hline
\end{tabular}

des. Algo que se evidencia si comparamos a Rubianes con Muros. En ambas, el cereal más cobrado es el trigo, en cambio, el segundo puesto ocupado en los totales respectivos, le corresponde en el primer caso a la cebada, mientras que en Muros el centeno ocupa el segundo lugar. La homogeneidad tampoco existe a la hora de analizar el cereal más recibido en cada administración. Finalmente, se percibe una clara diferenciación entre las administraciones castellanas, donde la mayor parte de la renta es recibida en dinero, y las gallegas, donde la amalgama de especies cobradas relega cualitativamente a un segundo puesto a aquélla. En definitiva una compleja variedad derivada de la distinta ubicación geográfica de las trece administraciones que componen el dominio, de las cuales nueve se sitúan en Galicia. 
Mapa de las administraciones del Dominio de Rubianes en Galicia.

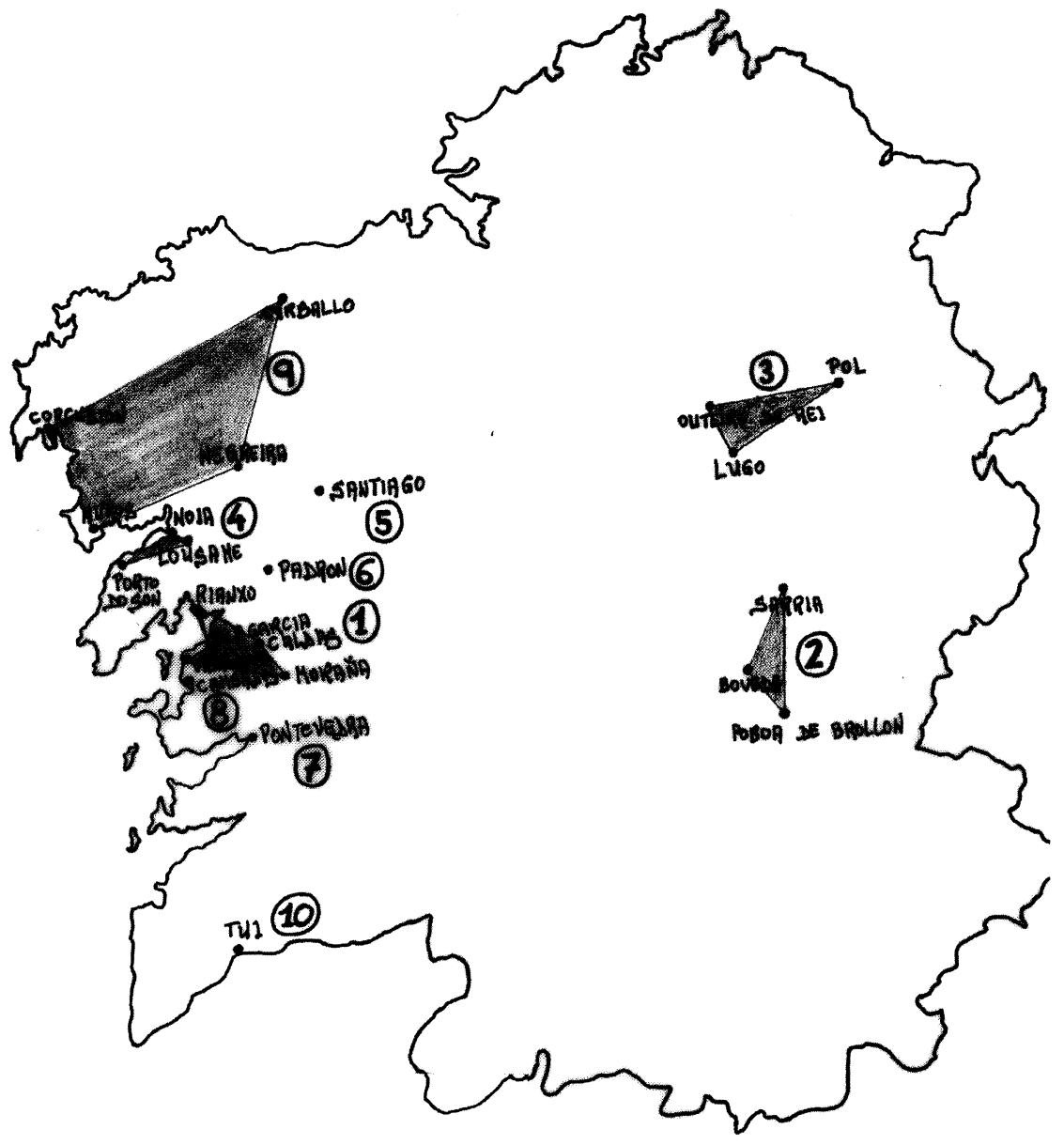
1. Rubianes
2. Guntín y Castelo dos Infantes
3. Lugo
4. Noia
5. Santiago
6. Padrón
7. Pontevedra
8. Cambados
9. Muros, Corcubión, Carballo y Negreira
10. Tui

"CUADERNOS DE ESTUDIOS GALLEGOS", Tomo XLII, Fascículo 107, Santiago 1995. 


\section{SOBRE LAS ESTRATEGIAS FAMILIARES A LA HORA DE REPARTIR EL MAYORAZGO}

La Ley de desvinculación de 1841 marca un punto y aparte respecto a lo que tenía acontecido anteriormente en el seno de los mayorazgos. A partir de entonces, el Estado liberal pretende que lleguen al mercado las tierras sujetas a los vínculos nobiliarios. Un proceso irreversible. Mas la agonía de la hidalguía gallega está caracterizada por un lento languidecer y adaptación a un nuevo orden de $\operatorname{cosas}^{25}$. Y los señores de Rubianes no se mantienen al margen.

Un año después de la muerte de Don Miguel acaecida en 1837, su señora viuda y su sobrino - Don José Ramón Ozores - firman un convenio $^{26}$. Se trata de una escritura de notable interés, pues en ella, los dos interesados deciden cómo se habrá de repartir el Dominio de Rubianes. Hay dos disposiciones del mismo que nos indican perfectamente cuál es la finalidad que les mueve a realizar tal convenio. Se especifica claramente que los bienes que conforman el Señorío de Rubianes tendrán que permanecer proindivisos sin que les afecte ninguna partija. Es decir, tanto Don José Ramón como su tía política Doña María del Pilar, poseerán cada uno su respectiva parte del patrimonio señorial, administrado conjuntamente, que habrá de permanecer sin dividir hasta la muerte de ella ${ }^{27}$. Por su parte, Doña María del Pilar podrá disponer de la casa-pazo de Rubianes $\mathrm{y}$ de sus anejos mientras viva, pero sin integrarla en el mercado ${ }^{28}$.

\footnotetext{
${ }^{25}$ R. Villares (1982b), pp. 141-150, M.J. Baz Vicente (1991), pp. 41-50, y L. Domínguez Castro (1992), pp. 115-125.

${ }^{26}$ AHUS, PN, ${ }^{\circ} 9.789,1838$, ff. 25-29.

${ }^{27} \mathrm{Tal}$ como se observa al leer Que sin perjuicio de la formalidad de las aplicadas posesiones, la Exma. Sra. otorgante desiste y se aparta de pedir en ningun tiempo desde su vida, la partija y division de la parte que le corresponde de los vienes vinculares, como de todos los libres y adquiridos en constante matrimonio, de que la ha instituido heredera el Sr. su difunto esposo en sus citadas disposiciones testamentarias, por ser su gusto y voluntad poseerlos, administrarlos y gozarlos sin necesidad de este tramite en union con el Exmo. Sr. otorgante..., AHUS, PN, $\mathrm{n}^{\circ}$ 9.789, f. 27.

${ }^{28}$ Septima: Manifiesta la Sra. constituyente que en prueba de su buen deseo del Sr. su esposo, la predicha Casa de Rubianes con su capilla, huerta y diestros del circumbalado llamado del Raposiño, con todos sus perfectos y mejoramientos, se impone asimismo la condición de cumplirlo sin poder vender, aforar ni de ninguna manera enagenar en propiedad lo mas minimo de todo ello..., AHUS, PN, $\mathrm{n}^{\circ}$ 9.789, 1838, f. 27.
}

"CUADERNOS DE ESTUDIOS GALLEGOS", Tomo XLII, Fascículo 107, Santiago 1995. 
Así, la señora viuda acepta como sucesor directo de su difunto esposo al señor de Rial - que es Don José Ramón-. Por lo tanto, el segundo señor de Rubianes de nuestra cuenta lo es a partir de 1838 , y posee a medias con su tía política un patrimonio que no podrá dividir hasta la muerte de aquélla. A partir de entonces, el señorío recaerá en los descendientes de Don José Ramón.

Pero el sobrino beneficiado muere en 1849 , dos años antes que Doña María del Pilar - que lo hace en 1851- Evidentemente, Don José Ramón era la mejor garantía para que el Señorío de Rubianes pasase a su hijo primogénito, Don Juan María, que se había casado con la marquesa de Aranda y Guimarey -Doña María Josefa Mosquera y Novales-. En el segundo testamento que hace Doña María del Pilar, del cual se incluye una copia en su partija, ratifica la disposición de dejarle sus bienes - es decir, su parte del señorío de Rubianes indivisible según los deseos iniciales de Don Miguel Gayoso, más los aportados por ella misma- a los descendientes de su difunto sobrino Don José Ramón ${ }^{29}$. Está implícita, pues, la idea de aferrar el patrimonio vinculado frente al nuevo orden de cosas en el que se favorece cada vez más su desmembración.

Don José Ramón es consciente del cambio que se está produciendo ${ }^{30}$. En su testamento se comprueba su preferencia por que su primogénito permanezca al frente del vínculo - tal como habría sucedido normalmen-

${ }^{29}$... y mediante no tengo herederos forzosos, es mi deliberada voluntad instituir como por el presente instituyo mi heredero unibersal en todos mis vienes, derechos y acciones que me corresponden y corresponder puedan, a su hijo, el Ecsmo. Sr. Dn. Juan Ozores Valderrama, actual señor de Rubianes, Grande de España de primera clase y Marques viudo de Aranda; y a la muerte de este, haya de pasar integra dicha mi herencia a su primogenito Dn. Jacobo, y subcesor en el titulo de señor de Rubianes..., AHUS, PN, $\mathrm{n}^{\circ}$ $10.242,1852$, f. 108.

${ }^{30}$ Es evidente a partir de la lectura detallada de su testamento: ... Quisiera que mi hacienda permaneciese unida como yo la disfrute y lo han hecho mis mayores, y a mi muerte pasase a su goce y usufructo mi hijo mayor e inmediato subcesor, porque al fin esta fue la voluntad absoluta y respetable de los fundadores; nació aquél con estos derechos, se educó vajo estas esperanzas y sobre ellas tomó estado, llegó a edad adulta y adquirió obligaciones sagradas, y que ahora llorará defraudadas; y que el indemnizase y recompensase luego de la manera mas conveniente, justa y equilibrada a sus otros hermanos en sus derechos y legitimas, pues yo a todos amo igualmente, y para todos como hijos de mi corazón deseo los mismos beneficios, la mejor y más ventajosa suerte; pero yo no puedo asi disponerlo porque las leyes vigentes ordenan otra cosa totalmente diversa..., AHUS, PN, $\mathrm{n}^{\circ} 10.240,1850$, f. 55.

"CUADERNOS DE ESTUDIOS GALLEGOS", Tomo XLII, Fascículo 107, Santiago 1995. 
te en el Antiguo Régimen-, después de recompensar debidamente a sus hermanos. Un sistema que garantizaba la solvencia económica de todos. Por una parte, el primogénito pasaba a ser el titular del vínculo al heredar la mayor parte de los bienes que lo componían. Y los hermanos, a su vez, recibían sus legítimas, obligándose a ello el primogénito mejorado.

Cuando este sistema entra en crisis con la aprobación de la ley desvinculadora de 1841, se sobreentiende que los antiguos señores intenten mantener la mayor parte de las posesiones que conforman el dominio unidas. Para ello se elige a la persona que haya de ser mejorada. En el caso de Rubianes, el primogénito, tal como se especifica en las mandas testamentarias de Don José Ramón Ozores. Según la nueva ley, se hace obligatoria la división del antiguo dominio en dos partes iguales, de las cuales una debía de pasar íntegramente al heredero principal, mientras que la segunda es susceptible de ser repartida entre el resto de los hermanos. Dentro de este marco, Don José Ramón beneficia claramente a su primogénito Don Juan María, ya que al margen de la mitad reservable de los bienes raíces heredados de su padre, que ascienden a 793.451 Rs., Don Juan María recibe la mejora del tercio de los bienes que se pueden dividir - 452.085 Rs.- , además de la legítima que debía recibir en igual cantidad que sus hermanos, que asciende a 90.417 Rs. Un total de 1.335.953 Rs. Su madre, Doña María Aurora Balderrama, recibe el quinto de la cantidad divisible, cifra que asciende a 324.148 Rs. El resto de los nueve hermanos tan sólo reciben cada uno de ellos su legítima de 90.417 Rs. Por lo tanto, una buena parte del patrimonio vinculado, por no decir la mayor parte del mismo, seguirá centralizado en la persona del varón heredero del título de señor de Rubianes ${ }^{31}$.

Esta panorámica se complica en la cuarta generación, en la que Don Jacobo sigue a su padre - Don Juan María Ozores - al frente del Señorío de Rubianes. Cuando mueren sus tías Doña María Manuela - 1858 - y Doña María de la Concepción - 1863 - , quien se beneficia en las disposiciones testamentarias de ambas es su abuela, Doña María Aurora Balderrama. Hay ciertos indicios que hablan de la aparición de un nuevo foco de interés en el seno de esta familia. De hecho, su tía Doña Manuela, además de beneficiar a Doña Aurora, le reserva a su hermana Doña Ramona

\footnotetext{
${ }^{31}$ Este primer paso parece similar al descrito por M.P. Sáinz de Rozas (1991) para el marquesado de Grañina; por otra parte, al marquesado de Lozoya, estudiado por A. García Sanz (1991), tan sólo se le aplicó una división patrimonial entre 1838 y 1844.
}

"CUADERNOS DE ESTUDIOS GALLEGOS", Tomo XLII, Fascículo 107, Santiago 1995. 
- y tras su muerte a su hija Doña Ramona de Prado- el quinto de sus bienes. Además, en la partija de los bienes de su madre - la marquesa de Aranda y Guimarey - Don Benito de Prado y Ozores - sobrino de Don Jacobo- recibe más cantidad que él por derechos que le pertenecen derivados de la legítima de su madre, Doña Concepción. Así, a mediados de la década de 1860, se destaca un nuevo elemento a tener en cuenta, como de hecho lo es el que ya no sólo sea el titular de la mayor parte de los bienes - en dicho momento Don Jacobo Ozores - el único beneficiario a la hora de redactar los distintos miembros de la familia sus respectivos testamentos.

Estos cambios experimentados a la altura de la segunda mitad del siglo XIX pueden ser indicadores del acercamiento de dos notables familias de la hidalguía gallega: los Prado, que son marqueses de San Martiño de Hombreiro, y los Ozores, señores de Rubianes, además de los títulos que fueron acumulando, tal como hemos visto.

\section{CONCLUSIONES}

Nos hallamos ante un dominio señorial perteneciente a la baja nobleza laica gallega que se ve afectado por las leyes desvinculadoras de principios del siglo XIX. Los miembros de la familia titular del Dominio de Rubianes se caracterizan por su absentismo geográfico respecto al núcleo principal del mismo, y sociológicamente, por su inclusión paulatina en el seno de profesiones liberales. Los segundones que antaño habían incrementado las filas del servicio doméstico, cuando no cogido los hábitos religiosos, pueden ahora optar por nuevas salidas dentro de un ámbito laboral que se complica a medida que avanza el siglo. El oficio de armas - como lo atestiguan Don Joaquín y Don Eduardo-, la política - destacándose Don José María como senador - y el Derecho - del cual Don José Javier es el mejor exponente- liberan a los miembros del grupo familiar nobiliario de las ataduras del pasado.

$\mathrm{Al}$ analizar pormenorizadamente los distintos dominios que componen el vínculo, se aprecia una clara diferenciación entre lo que acontece con los bienes sitos en Galicia, y aquéllos que lo están en Castilla. En ambos, de darse el caso de que los bienes patrimoniales sean objeto de arrendamientos y aforamientos a la vez, se observa como la renta derivada de los arriendos supera a la recibida de los foros. La mayor parte de la renta cobrada en dinero procede de arrendamientos hechos en las dos adminis-

"CUADERNOS DE ESTUDIOS GALLEGOS", Tomo XLII, Fascículo 107, Santiago 1995. 
traciones castellanas del vínculo - Badajoz y Zamora-, produciendo anualmente 81.742 Rs., que suponen algo más del $80 \%$ de la renta total en dinero. En Galicia, al contrario, la mayor parte de la renta se recibe en especie procedente de contratos de foro. También se recibe renta monetaria, pero casi siempre derivada del arriendo de inmuebles urbanos como queda palpablemente demostrado para la Administración de Santiago, donde los contratos de arrendamiento producen 2.153 Rs., en tanto los foros tan sólo 63 Rs. Con ello, es fácilmente deducible que el canon foral es cobrado principalmente en especie, y de manera mixta. No obstante, pese a la diversificación, se observa como en cada una de las distintas administraciones que componen el dominio, hay un producto cerealícola que se destaca por encima del resto. El trigo es mayoritario en Rubianes -39\% - , Santiago — 55,63\%—, Muros — 90,57\%—, y Cambados — $52,31 \%$ - si no contamos la renta procedente de los arriendos, ya que entonces, el primer puesto lo pasa a ocupar el maíz - 48\%-. El centeno se destaca como primer cereal cobrado en Guntín - 83,6\%- y Lugo - $90,86 \%$ - La cebada consigue alcanzar como máximo el $50 \%$ en la Administración de Pontevedra, en la que el 50\% restante se cobra en trigo; mientras que el mijo consigue también dicho porcentaje en Tui, convirtiéndose en mayoritario al dejar al centeno en $27 \%$, al trigo en $19 \%$ y al maíz en $4 \%$. De lo cual se deduce que nos hallamos ante una renta cerealícola basada en la preferencia por un cereal determinado que varía de una administración para otra.

Para finalizar, al detenernos en el estudio de las estrategias familiares, hemos podido demostrar que la mayor parte de los bienes que componen los distintos dominios del vínculo de Rubianes, permanecen unidos durante la primera mitad del siglo XIX, llegando así hasta 1865. Las distintas leyes desvinculadoras que acuña el liberalismo les afectan, pero tardan unos veinticinco años - los que van desde 1841 hasta 1865 - en hacerse sentir seriamente en los bienes del antiguo vínculo. La partija que hace Don José Ramón Ozores beneficiando a su hijo primogénito - Don Juan María - logra paliar los males que se derivan de la obligatoria puesta en el mercado de la mitad de los bienes vinculados, al mejorarlo con el tercio del remanente. En cambio, al llegar la cuarta generación, se intuye por parte de algunos miembros de la familia Ozores que redactan sus testamentos, que ya no benefician al primogénito. Posiblemente, la explicación se halle en la aparición de un nuevo centro de interés en el seno de la familia, al casarse Doña Ramona de Ozores con Don José de Prado -marqués de San Martiño de Hombreiro-.

"CUADERNOS DE ESTUDIOS GALLEGOS", Tomo XLII, Fascículo 107, Santiago 1995. 


\section{Anexo I. Cuadro genealógico de los Ozores del Señorío de Rubianes en el siglo XIX.}

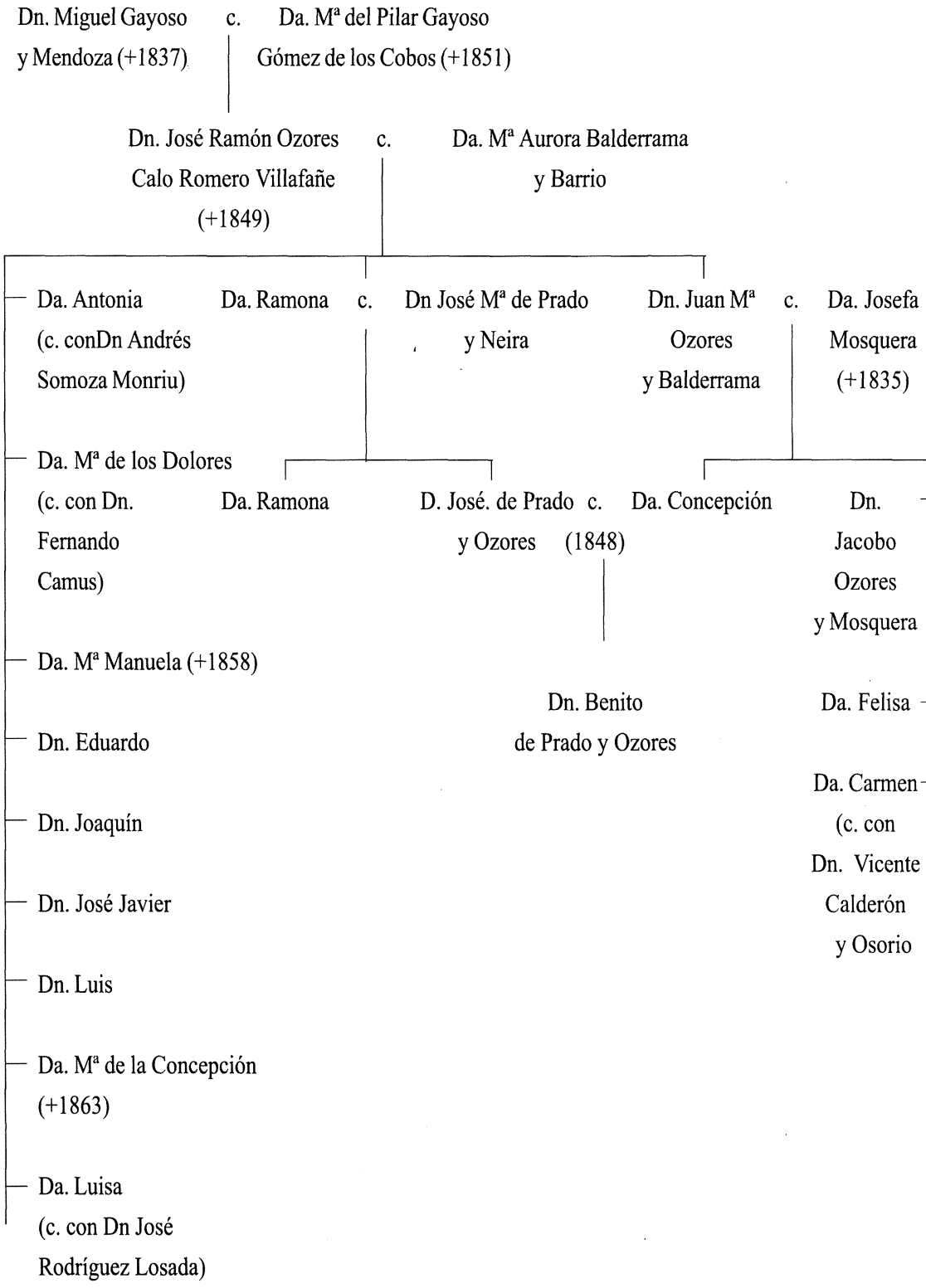




\section{BIBLIOGRAFÍA}

ARTIAGA, A. (1984): «La renta foral de Galicia a finales del siglo XIX», Agricultura y Sociedad, $\mathrm{n}^{\circ}$ 30, pp. 207-237.

ARTIAGA, A. et al. (1991): «Agricultura y capitalismo en Galicia: una perspectiva histórica», in. P. Saavedra y R. Villares (eds.): Señores y campesinos en la Península Ibérica, ss. XVIII-XX, vol. II, Barcelona.

BALBOA, X. (1990): O monte en Galicia, Vigo.

BAZ VICENTE, Ma J. (1991): El patrimonio de la Casa de Alba en Galicia en el siglo $X I X$, Lugo.

BERNÁRDEZ, A., CABO, M. Y PRESEDO, A. (1995): «Patrimonio familiar campesiño e cambio social na Galicia do s. XIX: propostas metodolóxicas», Historia Nova III, Santiago.

BOUHIER, A. (1979): La Galice. Essai géographique d'analyse et d'intrepretation d'un vieux complexe agrarie, La Roche-Sur-Yon.

CONGOST, R. (1990): Els propietaris i els altres, Vic.

CRESPO, J.S. (1958-85): Blasones y linajes de Galicia, Santiago.

DOMÍNGUEZ CASTRO, L. (1992): Viños, viñas e xentes do Ribeiro. Economía e patrimonio familiar, 1810-1952, Vigo.

GARCÍA SANZ, A. (1991): «Renta territorial y patrimonio de una casa nobiliaria en la Castilla del siglo XIX: los marqueses de Lozoya, 18081896», in P. Saavedra y R. Villares (eds.): Op. cit., vol. I, Barcelona.

LEIROS DE LA PEÑA, P. (1986): «La casa de Fontefiz (Contribución al estudio de la hidalguía gallega)», Santiago, tesis de licenciatura inédita.

MÍGUEZ RODRÍGUEZ, V.M. (1993): «Aproximación ao estudo da fidalguía galega a traverso do marquesado de San Martín de Ombreiro (1500-1800)», A Coruña, tesis de licenciatura inédita.

"CUADERNOS DE ESTUDIOS GALLEGOS", Tomo XLII, Fascículo 107, Santiago 1995. 
QUINTANA GARRIDO, X.R.: «Campesinos que se adaptan y agricultura que se mueve de la historia agraria de la Galicia contemporánea», Areas, $\mathrm{n}^{\circ} 12$, pp. 147-165.

RODRÍGUEZ DE LA HUERTA, J.M. y GÓMEZ ÁLVAREZ, U. (1992): Concejo de Caravia. Economía y Sociedad (1700-1750), Oviedo.

SAAVEDRA, P. (1982): «Los montes abiertos y los concejos rurales en Galicia en los siglos XVI-XVIII: aproximación a un problema», Cuadernos de Estudios Gallegos, t. 33, pp. 179-236.

- (1989): «La propiedad colectiva en Galicia en el siglo XVIII», in Estructuras agrarias y reformismo ilustrado en la España del siglo XVIII, Madrid.

- (1990): «Contribución al estudio del régimen señorial gallego», Anuario de Historia del Derecho Español, no 93, pp. 103-184.

SAINZ DE ROZAS, M.P. (1991): «La pervivencia de las estrategias vinculares en las transmisiones testamentarias de la segunda mitad del siglo XIX. El caso del marquesado de Grañina, 1850-1875», in P. Saavedra y R. Villares (eds.): Op. cit., vol. I, Barcelona.

SUAU PUIG, J. (1991): «Estudio sobre la renta de la tierra. Mallorca, 1750-1860», in P. Saavedra y R. Villares (eds.): Op. cit., vol. I, Barcelona.

VILLARES, R. (1982a): Foros, frades e fidalgos, Vigo.

- (1982b): La propiedad de la tierra en Galicia, 1500-1936, Madrid.

YUN CASALILLA, B. (1991): «Vasallos y señores en el marquesado de Cuéllar: relaciones sociales, aprovechamiento de recursos y gestión señorial a fines del Antiguo Régimen», in. P. Saavedra y R. Villares (eds.): Op. cit., vol. I, Barcelona. 